\title{
Structural study of phenylperimidines with potential applications as corrosion inhibitors
}

\author{
E Olave Negrete ${ }^{1}$, M Espinoza $^{1}$, V Artigas ${ }^{1}, \mathbf{R}$ Sánchez ${ }^{1}, \mathbf{R}$ Vera ${ }^{1}$, M Fuentealba $^{1}, \mathrm{~J}$ Aristizabal ${ }^{2}$ \\ ${ }^{1}$ Pontificia Universidad Católica de Valparaíso, Valparaíso, Valparaíso, ${ }^{3}$ Universidad Técnica \\ Federico Santa María, Valparaíso, Valparaíso \\ elizabeth.olave@pucv.cl
}

Worldwide the protection against the corrosion is mainly based on the use of corrosion inhibitors; they exist in a variety of organic and inorganic compounds added on the metal surface. An inhibitor must possess structural features, such as having a planar geometry, $\pi$-conjugated system, bearing functional groups and heteroatoms with unshared electron pairs. With these characteristics, corrosion inhibitors will generate a barrier against corrosion through physisorption and chemisorption processes.

In this work, we carried out the syntheses and characterization, using vibrational spectroscopy, of phenylperimidines compounds synthesized from 1,8-diaminonaphtalene and 2-hydroxybenzaldehyde. The condensation reaction is carried out by refluxing the reactants in toluene obtaining the 2-(1H-perimidin-2-yl) phenol (a) compound and, in toluene/methanol 2: 1 mixture, the 2-(2,3-Dihydro -1H-perimidin-2-yl) -phenol (b) is formed (Fig. 1). The crystal structures of both compounds (a) and (b) has been determined by X-ray single-crystal diffraction. From the molecular and crystalline structures, it is observed that the structure of (a) only crystallizes with one molecule in the asymmetric unit forming an intramolecular hydrogen bond $\mathrm{O} 1-\mathrm{H} 1 \cdots \mathrm{N} 1$ granting planarity to the system. On the other hand, the compound (b) crystallizes with two formula units in the asymmetric unit. In this case, an intramolecular hydrogen bond is formed between the $\mathrm{O} 1-\mathrm{H} 1 \cdots \mathrm{N} 1$ and the $\mathrm{O} 1 \mathrm{~A}-\mathrm{H} 1 \mathrm{~A} \cdots \mathrm{N} 2 \mathrm{~A}$ atoms.

Both compounds are postulated as potential corrosion inhibitors, since the crystalline and molecular structures of (a) and (b) present intra- and intermolecular hydrogen bonds, have functional groups bearing heteroatoms and contain a $\pi$-conjugated system in (a) that yields to a flat geometry with high electronic communication unlike (b) that loses planarity over $\mathrm{C} 11$ with $\mathrm{sp} 3$ hybridization. The cyclic voltamperometry measurement of (a) is reported (Fig. 2). The oxidation process is observed with a potential peak defined in $+0.328 \mathrm{~V}$ and the reduction processes with two waves at $+0.106 \mathrm{~V}$ and $-0.346 \mathrm{~V}$. Also, there are no drastic changes in the different cycles of voltammetry, indicating the structural stability of the compounds. Therefore, these compounds could be used as a corrosion inhibitor by the conjugation of aromatic rings capable of interacting with the metal surface in a specific medium and metal or alloy for study and simulation.

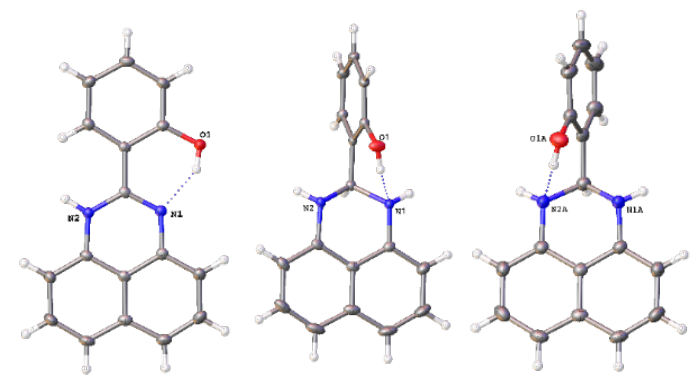

(a) (b)

Figure 1. The ORTEP diagrams with $30 \%$ ellipsoidal probability of 2-(1H-perimidin-2-yl) phenol (a) and 2-(2,3Dihydro -1H-perimidin-2-yl) -phenol (b)

Acta Cryst. (2020). A76, a187-a188 


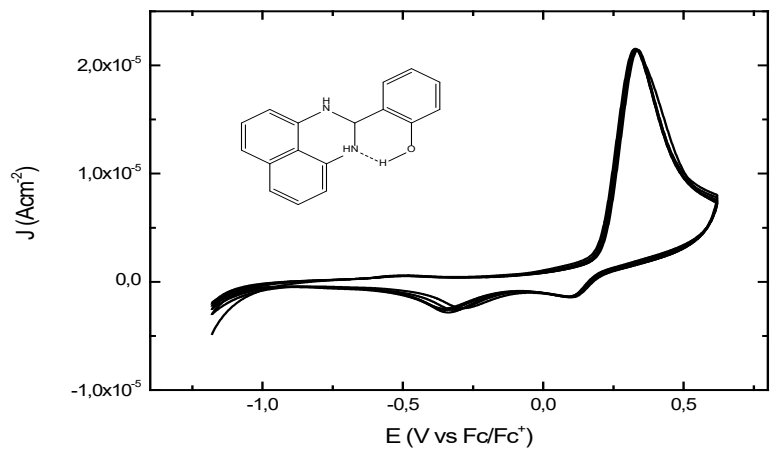

Figure 2. Cyclic voltamperogram of 2-(1H-perimidin-2-yl) phenol (a) between $-1.5 \mathrm{~V}$ and $+0.6 \mathrm{~V}$ at $50 \mathrm{mV} \mathrm{s}-1$.

Acta Cryst. (2020). A76, a187-a188 